\title{
Diabetes Mellitus in the Middle-Aged and Elderly Population (>45 Years) and Its Association With Pancreatic Cancer: An Updated Review
}

\author{
Thanmai Kaleru $^{1}$, Varun K. Vankeshwaram ${ }^{2}$, Ankush Maheshwary ${ }^{3,4}$, Divya Mohite ${ }^{3}$, Safeera Khan ${ }^{5}$ \\ 1. Internal Medicine, California Institute of Behavioral Neurosciences and Psychology, Fairfield, USA 2. Medicine, \\ Zaporozhye State Medical University, Zaporozhye, UKR 3. Neurology, California Institute of Behavioral Neurosciences \\ and Psychology, Fairfield, USA 4. Medicine, Government Medical College, Amritsar, IND 5. Internal Medicine, \\ California Institute of Behavioral Neurosciences \& Psychology, Fairfield, USA
}

Corresponding author: Thanmai Kaleru, thanmaikaleru3@gmail.com

\begin{abstract}
Diabetes mellitus (DM) and pancreatic cancer (PC) in the elderly are widely considered to be interrelated. New-onset diabetes (NOD) patients are considered a high-risk group for the development of PC within three years of diagnosis. We reviewed the literature to determine the pathophysiological association between DM and PC, which can help in the development of screening tests for early PC diagnosis in the elderly with NOD. We also studied the potential associations between them after pancreaticoduodenectomy (PD) or pancreatic resection. We collected studies published in the last five years in PubMed that are relevant to DM and PC in the elderly. We mainly focused on the pathophysiology and intracellular mechanisms involved between NOD and PC. We illustrated the clinical signs and immunological and metabolic biomarkers that can be used to diagnose early PC in the elderly with NOD. In the 34 studies we reviewed, five showed that long-term diabetes mellitus (LTDM) increases the risk of PC. Six studies showed that NOD in the elderly is an early sign of PC. Fourteen studies proposed that clinical signs and biomarker levels should be used to determine the high-risk risk group for PC among NOD patients. Six studies reported that NOD is associated with the worst outcomes postoperatively, and three studies showed that patients developed DM after pancreatic resection. LTDM is considered an independent risk factor for PC development in the elderly. NOD is a consequence and maybe the only early presenting sign of PC. Screening protocols and tests should be used in clinical practice to determine the proportion of NOD patients who should undergo further testing for early diagnosis of PC. DM and PC are also co-related postoperatively and patients should be monitored for impaired glucose levels, overall survival, and mortality.
\end{abstract}

Received 06/03/2020

Review began 06/22/2020 Review ended 06/23/2020 Published 06/28/2020

() Copyright 2020 Kaleru et al. This is an open access article distributed under the terms of the Creative Commons Attribution License CC-BY 4.0., which permits unrestricted use, distribution, and reproduction in any medium, provided the original author and source are credited.
Categories: Endocrinology/Diabetes/Metabolism, Internal Medicine, Oncology Keywords: diabetes mellitus in elderly, new-onset diabetes, pancreatic cancer

\section{Introduction And Background}

Diabetes Mellitus (DM) is a complex metabolic disorder with characteristic hyperglycemia that arises either due to defective insulin secretion by pancreatic islets or due to insulin resistance of peripheral tissues or deregulated hepatic glucose production. Diagnostic criteria is defined as two consecutive readings of fasting blood glucose (FBG) of >126 mg/dl; two-hour post-glucose level of >200 mg/dl (oral glucose tolerance test); random blood glucose level of $>200 \mathrm{mg} / \mathrm{dl}$; or glycated hemoglobin (HbA1c) level of $>6.5 \%$ [1]. The global prevalence of diabetes as reported by the International Diabetes Federation in 2019 was $9.3 \%$, and it is estimated to rise to $10.2 \%$ by 2030 [2].

DM manifests as either type 1 (due to beta cell destruction, leading to absolute insulin deficiency) or type 2 (predominantly due to insulin resistance with relative insulin deficiency or insulin secretory defect). Other, less common types of diabetes are as follows: those associated with genetic defects of beta cells or insulin action, drug and chemical-induced diabetes, endocrinopathies, and disease of the exocrine pancreas (type $3 \mathrm{c}$ diabetes). In this review, we considered new-onset diabetes (NOD) in the last two to three years as a diagnosis of DM in patients with an FBG of $>126 \mathrm{mg} / \mathrm{dL}$ or HbA1c of $>6.5 \%$ without a known history of diabetes. Long-term diabetes mellitus (LTDM) was defined as a diagnosis of DM made for more than three years. Typically, type 1 diabetes is diagnosed among younger patients. On the other hand, type 2 DM caused by insulin resistance is the most common type seen in older populations. In this article, DM is referred to as type 2 diabetes by default. The average range of peak age of diagnosis of type $2 \mathrm{DM}$ is considered to be between 45-60 years [3]. Another study published by the American Diabetes Association (ADA) states that type $3 \mathrm{c}$ diabetes (diabetes following pancreatic disease) often misdiagnosed as type $2 \mathrm{DM}$ is associated with poor glycemic control and has a higher incidence than type $1 \mathrm{DM}$, with a peak age of 59 years [4]. According to the Centers for Disease Control and Prevention (CDC) reports, the incidence of any type of diabetes increases with age and tends to stem off after the age of 65 years [2]. Long-term diabetes is prevalent in the elderly and is a risk factor for all malignancies, including pancreatic cancer (PC) [5]. There are many risk factors (smoking, age, alcohol, chronic pancreatitis, diabetes, obesity, genetic, and other cancer syndromes) contributing to the development of PC [6]. Many studies have documented a positive association between 
LTDM and PC $[5,7,8,9]$. This can be explained by the risk factors (age, obesity, genetic factors, and lifestyle changes) or pathophysiology (impaired action of the insulin) shared by the two diseases. NOD in the elderly is considered to be highly associated with PC, with reported evidence of nearly $40 \%$ of patients diagnosed with NOD developing PC within three years of diagnosis [10,11].

PC is less prevalent, yet it is one of the deadly malignancies with high death rates [12]. According to 2019 cancer statistics, it is the tenth most common type of cancer among women and ninth in men, but the fourth leading cause of cancer-related deaths among all the cancers in the US [13]. Sporadic PC is more prevalent in individuals who are more than 50 years of age with NOD [14,15]. Pancreatic adenocarcinoma (PAC), arising from the pancreatic ductal cells, is the most common type of PC and accounts for $90 \%$ of all PC cases. In this review, we have referred to PAC as PC. Most patients with PC are asymptomatic or have vague and unexplained symptoms like pain, weight loss, jaundice, loss of appetite, nausea, pancreatitis, and new onset of diabetes [16]. Imaging tests (CT, MRI, and endoscopic ultrasound) are the main screening modalities used for the diagnosis of PC. However, due to the low incidence and the early asymptomatic phase of the tumor, population-based screening is not practiced in the clinical setting. Hence, the majority (85\%) of the patients at the time of diagnosis are in their advanced stages and are not eligible for surgical resection $[14,17]$. Thus, early screenings have a significant impact on the prognosis of this aggressive tumor. We performed a literature review of the articles explaining the association between the two diseases and reported the potential pathophysiological and intracellular mechanisms involved.

Early and effective detection programs in the past decade have improved the treatment options and prognosis and reduced the mortality rates of other cancers [15]. However, in the case of PC, the advances have been minimal due to the uncertainty regarding the proportion of patients to be tested. Elderly patients with NOD represent a high-risk group and should be potentially screened for early diagnosis of PC. Proposed clinical models and interventional strategies may help in early diagnosis [15]. However, there is still a lack of clarity regarding standard criteria and diagnostic tests that differentiate NOD from NOD-PC (new-onset diabetes with pancreatic cancer). In this review, we discuss the various clinical signs and immunological and metabolic biomarkers that have proven useful in distinguishing the subpopulation of NOD-PC from NOD in the elderly. We also discuss the clinical outcomes in terms of glucose levels and prognosis of PC postsurgery.

\section{Review}

\section{Methods}

We followed the preferred reporting items for systematic review and meta-analysis (PRISMA) guidelines (Figure 1) and relied mainly on PubMed to search for articles that are relevant to diabetes and PC and explain the association between these two diseases. We conducted the literature search by using the combination of keywords "diabetes mellitus in elderly," "new-onset diabetes," and "pancreatic cancer."

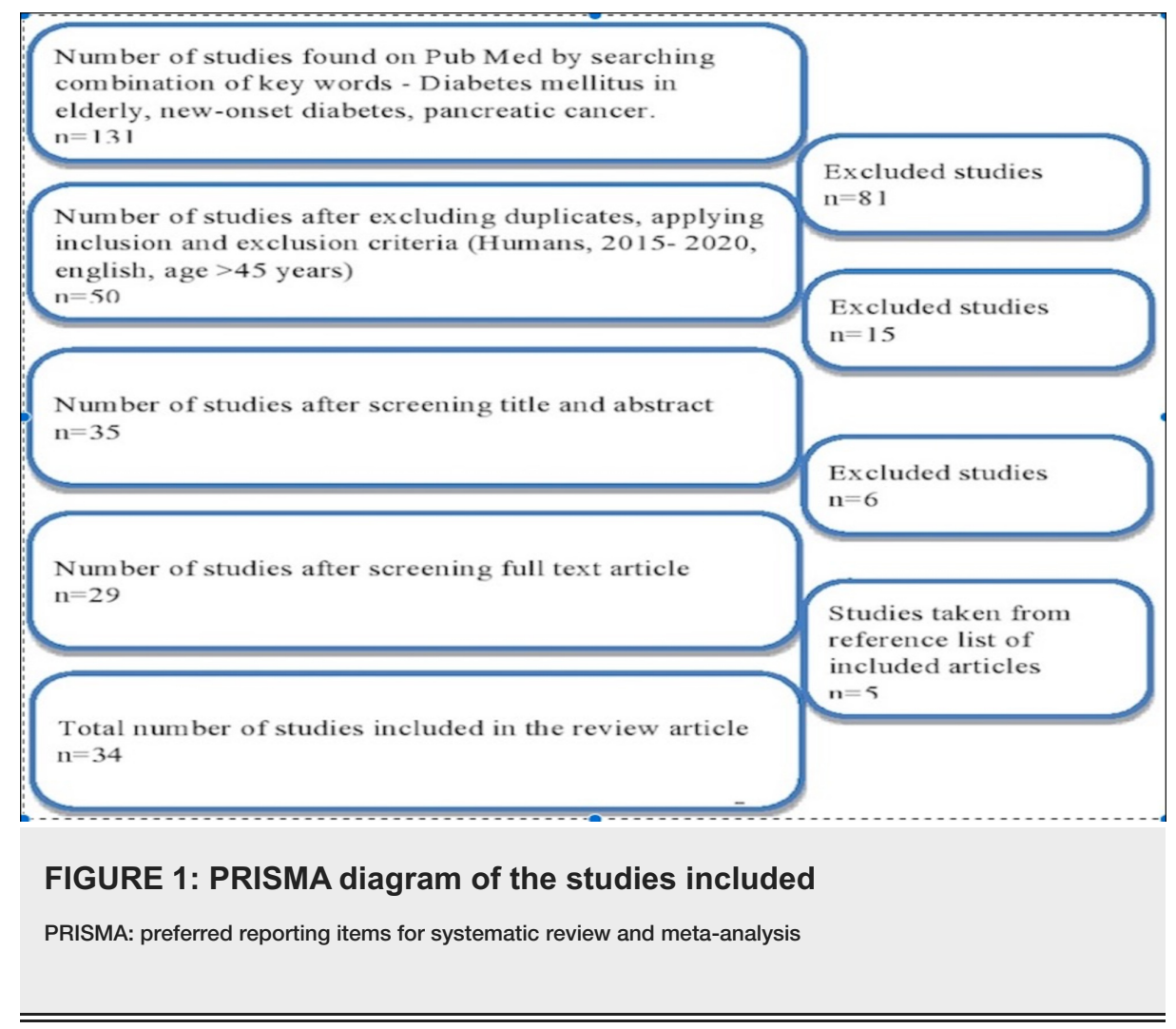


We included peer-reviewed studies that are relevant to the topic. All the studies that are included were published in the English language within the last five years before the search date (2015-2020). We did not focus on any specific geographical location for the study search. However, we limited our search to the middle-aged and elderly population ( $>45$ years). Our study results included both the abstract and full-text articles and did not include any form of grey literature.

We focused on the potential pathophysiology and intracellular mechanisms involved between NOD and PAC. We excluded the mechanisms and associations involved in other types of PC. We particularly focused on changes in the clinical signs and immunological and metabolic biomarkers that can be used to diagnose early PC in the elderly with NOD.

All data were collected in an ethical and legal manner. Quality appraisal of the studies included was done using the Cochrane risk-of-bias tool for randomized trials, Newcastle-Ottawa Scale for observational studies, and the assessment of multiple systematic reviews (AMSTAR) checklists for systematic reviews/metaanalyses.

\section{Results}

By following the search criteria mentioned in the methods section, we collected a total of 50 articles that are relevant to NOD and PC. We further filtered the relevant articles by reading the title, abstract, and full text to a final number of 34 articles included in this review. Among them, six are randomized control trials, 19 are observational studies, one is a systemic review, and three are meta-analyses. The remaining three are clinical guidelines and other publications. All of the studies included are peer-reviewed.

Among the included articles, four studies explained that LTDM is an independent risk factor for PC $[5,7,8,9]$. Seven studies showed that NOD precedes the onset of PC [9-12,18-20], and 13 studies proposed as to how NOD with certain clinical factors and screening tests can be used as a predictor for PC screening $[1,7,12,14,16,18,19,21-27]$. Seven studies mentioned the relation between PC and DM after pancreatic surgery [28-34]. Details of a few of the important studies are summarized in Table 1. 


\section{Cureus}

\begin{tabular}{|c|c|c|c|c|}
\hline $\begin{array}{l}\text { Author } \\
\text { name and } \\
\text { year of } \\
\text { publication }\end{array}$ & $\begin{array}{l}\text { Type of } \\
\text { study and } \\
\text { number of } \\
\text { subjects } \\
\text { included (n) }\end{array}$ & Study purpose & Results & Conclusion \\
\hline $\begin{array}{l}\text { Sharma et } \\
\text { al., } 2018 \\
\text { [14] }\end{array}$ & $\begin{array}{l}\text { Observational } \\
\text { cohort study; } \\
n=1,561\end{array}$ & $\begin{array}{l}\text { To develop a model to } \\
\text { determine the risk of } \\
\text { PC in NOD patients }\end{array}$ & $\begin{array}{l}\text { Patients with an END-PAC score of zero has } \\
\text { an extremely low risk of PC. A score of }>3 \\
\text { identified } 75 \% \text { of patients with six months' } \\
\text { early diagnosis of PC }\end{array}$ & $\begin{array}{l}\text { Change in weight, blood } \\
\text { glucose, and age at onset of } \\
\text { diabetes can be used as a } \\
\text { model (END-PAC) to contribute } \\
\text { to early detection of PC }\end{array}$ \\
\hline $\begin{array}{l}\text { Choe et al., } \\
2018 \text { [16] }\end{array}$ & $\begin{array}{l}\text { Observational } \\
\text { study; } \\
\mathrm{n}=5,111\end{array}$ & $\begin{array}{l}\text { To assess the utility of } \\
\text { CA } 19-9 \text { as a } \\
\text { screening indicator of } \\
\text { PC with } \\
\text { asymptomatic } \\
\text { patients with NOD }\end{array}$ & $\begin{array}{l}\mathrm{PC} \text { is detected in } 3.8 \% \text { of patients with } \\
\text { normal bilirubin levels and high CA } 19-9 \\
\text { levels and } 0.3 \% \text { with normal CA } 19-9 \text { levels }\end{array}$ & $\begin{array}{l}\text { CA } 19-9 \text { can be a useful } \\
\text { biomarker of PC in patients } \\
\text { after diagnosis of NOD }\end{array}$ \\
\hline $\begin{array}{l}\text { Mueller et } \\
\text { al., } 2019 \\
\text { [21] }\end{array}$ & $\begin{array}{l}\text { Case-control } \\
\text { study }\end{array}$ & $\begin{array}{l}\text { To evaluate blood } \\
\text { glucose levels and } \\
\text { weight change as } \\
\text { predictors of PC in } \\
\text { NOD }\end{array}$ & $\begin{array}{l}\text { Weight loss of }>15 \% \text { is associated with an } \\
\text { OR of } 4.56 \text { compared to a stable weight, and } \\
\mathrm{HbA} 1 \mathrm{c} \text { of }<5.1 \mathrm{mmol} / \mathrm{L} \text { before two to three } \\
\text { years of cancer has OR of } 2.42 \text { compared to } \\
\mathrm{HbA} 1 \mathrm{c} \text { of }>6.3 \mathrm{mmol} / \mathrm{L}\end{array}$ & $\begin{array}{l}\text { Normal weight and blood } \\
\text { glucose levels before diabetes } \\
\text { onset may be predictive of } \\
\text { NOD-PC }\end{array}$ \\
\hline $\begin{array}{l}\text { Lee et al., } \\
2018 \text { [29] }\end{array}$ & $\begin{array}{l}\text { Observational } \\
\text { study }\end{array}$ & $\begin{array}{l}\text { To analyze clinical } \\
\text { outcomes of PC with } \\
\text { NOD }\end{array}$ & $\begin{array}{l}\text { Overall survival of patients with NOD was } \\
\text { worse than non-DM } 22 \text { vs. } 33 \text { months }\end{array}$ & $\begin{array}{l}\text { NOD represents aggressive } \\
\text { tumor biology of PC }\end{array}$ \\
\hline $\begin{array}{l}\text { Song et al., } \\
2015 \text { [5] }\end{array}$ & $\begin{array}{l}\text { Meta- } \\
\text { analysis }\end{array}$ & $\begin{array}{l}\text { To determine if LTDM } \\
\text { is an independent risk } \\
\text { factor for PC }\end{array}$ & $\begin{array}{l}\text { RR of PC with a duration of diabetes of }>2 \\
\text { years, }>5 \text { years, and }>10 \text { years was } 1.64, \\
1.58,1.50 \text { respectively }\end{array}$ & $\begin{array}{l}\text { LTDM (>2 years) is associated } \\
\text { with an increased risk of PC } \\
\text { but negatively correlated with } \\
\text { the duration }\end{array}$ \\
\hline
\end{tabular}

\section{TABLE 1: Description of selected studies included in the review}

LTDM: long-term diabetes mellitus; PC: pancreatic cancer; NOD: new-onset diabetes mellitus; END-PAC: enriching new-onset diabetes for pancreatic cancer; CA 19-9: carbohydrate antigen 19-9; NOD-PC: new-onset diabetes with pancreatic cancer; RR: relative risk; OR: odds ratio; HbA1c: glycated hemoglobin

\section{Discussion}

There is a bidirectional relationship between DM and PC. Overall, DM is associated with a two-fold increased risk of PC. When it is further stratified according to the duration of onset, NOD ( $<3$ years) is associated with a 1.5-1.7 fold increase in the risk of PC [12,5]. LTDM is considered an independent risk factor for PC and, on the other hand, NOD is considered an early manifestation. The pathophysiological mechanisms involved in this relation are complicated and are explained in Figure 2. To elucidate the underlying pathophysiology and propose a feasible PC screening strategy for early diagnosis among the high-risk groups, we interpreted and analyzed various studies that explained the potential mechanisms, screening models, and tests.
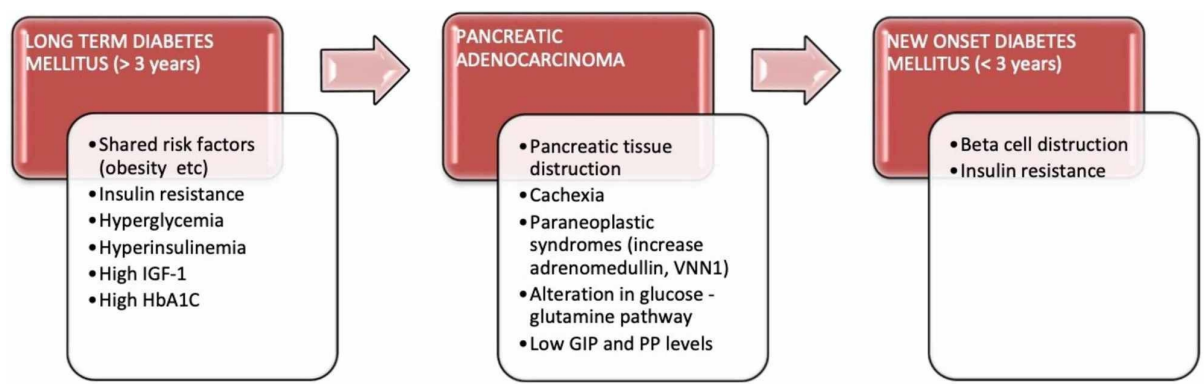

FIGURE 2: Pathophysiology of the association between diabetes mellitus and pancreatic cancer 
A meta-analysis published in 2015 showed that diabetes lasting more than two years is considered as an independent risk factor for PC [5]. The likely mechanism explained for this increased risk of carcinogenesis is related to the simultaneous increase in both the insulin-like growth factor-1 (IGF-1) levels and insulin signaling pathways, which causes hyperglycemia, insulin resistance, and hyperinsulinemia. Increased IGF-1 levels cause tumor pathogenesis by inducing the proliferation and inhibition of apoptosis in the target tissues. Hyperglycemia can also cause oxidative stress in the tissues by increasing the reactive oxygen species. However, it is not clear if it is the above pathogenesis or the shared risk factors like obesity that play a major role in an increased incidence of PC in patients with DM. Another pooled analysis conducted by McWilliams et al. in 2016 tested the risk factors for very early ( $<45$ years age) and early (age less than 60 years) onset of PC and proposed that LTDM appeared to be more effective in the development of PC in older ages ( $>45$ years) compared to younger patients ( $<45$ years) [9]. However, the difference in the risk between the two age groups was not fully explained. High HbA1c levels at the onset of diabetes further correlate with a higher incidence of PC [8]. However, this correlation was only explained in patients with NOD ( $<3$ years). On the other hand, complications of LTDM like kidney failure and high serum creatine are inversely related to PC development [7]. This could be due to the high mortality rate in diabetics with these complications. There is limited literature available on the role of anti-diabetic medications in the development of PC. However, some studies suggested that the use of metformin is associated with minimal risk or protective against PC, and the use of insulin is associated with the highest risk [8]. From all these studies, it is evident that prolonged increased levels of glucose and insulin are the main components that lead to carcinogenesis.

Significantly, $73 \%$ of patients with PC were assessed to have recently diagnosed with DM or pre-diabetes within two years retrospectively [10]. This type of cancer-related NOD is highly associated with the elderly because the prevalence of PC increases and NOD decreases after the age of 65 years [9]. Investigation of underlying mechanisms of PC-induced NOD might help to come up with the potential strategies for early screening, diagnosis, and treatment. Two potential phenomena explained previously are progressive pancreatic tissue destruction by tumor growth and cachexia, and the other is by the development of insulin resistance as one of the paraneoplastic syndromes caused by tumor secreting products [11]. However, the size of the tumor is not directly proportional to the blood glucose levels. It is further explained by high glucose levels in the early stages when the size of the tumor is very small for detection. Another study published earlier argued that the loss of beta-cell function through hormonal mechanisms plays a major role compared to insulin resistance in the development of NOD in PC [18]. It suggested that increased expression of proteases results in decreased levels of selective gastrointestinal hormones like glucose-dependent insulinotropic peptide (GIP), pancreatic polypeptide (PP), and a further decrease in insulin secretion from beta cells. High adrenomedullin levels are found in NOD-PC compared to NOD alone [12]. There is also overexpression of vanin-1 (VNN1), and its downstream metabolic products are observed in PC [19]. These were found to exacerbate paraneoplastic diabetes through the synergetic damage to the islet cells by triggering oxidative stress within the pancreatic microenvironment. Alterations in the circulating levels of several metabolites of amino acids, glucose and glutamine pathways, bile acid, and sphingolipids have been observed in NOD-PC [20]. Detection of these biomarker levels can help to stratify the subpopulation of highrisk patients from the NOD cohort that could be referred for screening with imaging. However, these were observational findings, and further interventional studies are required to validate the significance of using them in PC screening. A different pathway of cancer-genesis without affecting the glucose metabolism is explained by high microRNA (mRNA) levels; mainly, a significant increase in mRNA-200 and mRNA-192 in severe and progressive PC is observed [10]. Measuring their levels might help in cases of susceptible highrisk patients with normal glucose levels.

Screening Strategies for Early Diagnosis of PC in Patients with NOD

NOD could be the earliest sign of PC and patients with NOD are considered as a high-risk group for earlystage, asymptomatic cancer. A sudden increase in the blood glucose levels in diabetics who were well controlled previously may also be a sign of PC. However, screening all the elderly NOD patients with imaging tests for PC is challenging in the clinical practice and not cost-effective. Several clinical strategies have been proposed that might be helpful as an initial filter to stratify individuals that can be referred for further screening. A retrospective, population-based cohort study conducted in the United Kingdom assessed clinical parameters like age, BMI, change in the BMI, HbA1c levels, anti-diabetic medications, and other factors and developed the risk model that stated that only $6.19 \%$ would undergo and benefit from the definitive screening of PC if the risk threshold for PC within three years was set at 1\% [7]. However, this model also included the younger age groups ( $>35)$ compared to the individuals that are the focus of the current review article $(>45)$. It also has to be validated externally before it can be considered in clinical use. Another prospective observational cohort study initiated by the Consortium for the Study of Chronic Pancreatitis, Diabetes, and Pancreatic Cancer (CPDPC) proposed a new approach (define, enrich, find) to define and enrich the high-risk group of PC and find the lesion in the high-risk cohort [1]. The use of 
enriching new-onset diabetes for pancreatic cancer (END-PAC) score to stratify subjects of $>50$ years into high, intermediate, and low-risk groups can also be an effective tool for clinicians to proceed for further screening [14]. Older patients with weight loss (low BMI) and a rapid increase in glucose levels in a short duration are predictive of $\mathrm{PC}$ and could be the target population for further screening [21]. Other useful differentiating factors predicted to be tested to differentiate NOD from NOD-PC are BMI, age of onset, hepatitis B virus (HBV), total bilirubin (TBIL), alanine aminotransferase (ALT), creatinine (Cr), apolipoprotein-A1 (APO-A1), and white blood cells (WBC) [22]. Fatigue and depression caused by elevated cytokine production, interleukin 6 (IL-6), along with severe weight loss (>10\%) and NOD can be early paraneoplastic signs and potential precursors for PC [23]. A prediction model of medicare enrollees provided some information about the risk factors that could be used to test for emergent diagnosis of PC. However, it was not applicable for population screening since they have excluded the data for three months before the development of PC [24]. All the above studies are to be validated further by conducting population-based prospective interventional studies to find out the best strategy. However, clinical implementation of these strategies may reduce the dilemma regarding PC screening in NOD patients.

Many other studies have discussed the potential screening tests for early diagnosis of PC. Carbohydrate antigen (CA) 19-9 level excreted by cancer cells is a well-established indicator of PC with NOD compared to asymptomatic PC patients. However, it has a high false-positive rate as it is increased in any condition that leads to inflammation of the pancreas. A study has recommended assessing the CA 19-9 and liver function tests (LFT) in NOD patients seen in clinical settings [16]. An earlier study revealed that CA 19-9 levels are not significant in screening PC in NOD as both positive predictive value (PPV) and sensitivity were zero and had a false positive rate (FPR) of 9\% [25]. But this was tested in a small number of cases and cannot be applied to the population. The same study also mentioned that CT was a reliable screening method compared to the ultrasound. However, the risk of radiation exposure should be weighed over its benefits, and other easy, noninvasive screening tests should be used in the first stages of clinical encounter. High levels of bilirubin are considered a more effective test for tumors arising from the head of the pancreas as it correlates with PC development even with CA 19-9 levels being normal (<37 U/ml). Therefore, CA 19-9 could be considered as a better screening test with normal bilirubin levels, which helps in detecting PC arising from the tail of the pancreas. A later study also emphasizes that CA 19-9 could be used as a cost-effective test in screening early PC lesions in the first two years of NOD development, which are too small to be detected on imaging studies [26]. This helps to alert the clinicians for regular monitoring of PC in NOD patients even with negative imaging results. Alterations in levels of metabolites of primary bile acid synthesis, sphingolipid, and amino acid metabolism are also observed in cases with NOD-PC compared to controls of NOD patients [20]. Studies have shown that tumor-secreted products like adrenomedullin, mRNA panel, VNN1 and its downstream molecules, and gastrointestinal hormones like GIP and PP can be used as the biomarkers potentially useful in distinguishing type 2 DM from DM with PC $[12,27,19,18]$. However, a more comprehensive panel of the tumor-secreted diabetogenic factors that result in the paraneoplastic development of NOD should be used to implement screening along with clinical characteristics and CA 19-9 levels. The clinical and biological factors that help to distinguish between NOD-PC and NOD are briefly explained below (Table 2).

\begin{tabular}{|c|c|c|}
\hline $\begin{array}{l}\text { Clinical } \\
\text { signs/biomarkers }\end{array}$ & NOD with pancreatic cancer (NOD-PC) & NOD without pancreatic cancer (NOD) \\
\hline Patient age & $>65$ years & $<45$ years \\
\hline $\begin{array}{l}\text { Change in } \\
\text { weight/BMI }\end{array}$ & Rapid weight loss/low BMI & A gradual loss of weight/high BMI \\
\hline $\begin{array}{l}\text { Change in } \\
\text { glucose levels }\end{array}$ & $\begin{array}{l}\text { A rapid change in a short period of time, higher } \\
\text { HbA1c, higher glucose levels }\end{array}$ & $\begin{array}{l}\text { A gradual change in a longer duration of time, lower } \\
\text { HbA1c, lower glucose levels }\end{array}$ \\
\hline Adrenomedullin & Increased & Normal \\
\hline $\begin{array}{l}\text { VNN1 and } \\
\text { metabolites }\end{array}$ & Increased & Normal \\
\hline Micro RNAs & Increased & Normal \\
\hline Metabolomics & Increased & Normal \\
\hline GIP, PP & Decreased & Normal \\
\hline
\end{tabular}

\section{TABLE 2: Distinguishing clinical and biological factors between NOD-PC and NOD}

NOD: new-onset diabetes; NOD-PC: new-onset diabetes with pancreatic cancer; BMI: body mass index; VNN1: vanin-1; GIP: glucose-dependent insulinotropic peptide; PP: pancreatic polypeptide 
Surgical resection or pancreatoduodenectomy (PD) is the most effective treatment for PC compared to chemotherapy or other treatment options. Even though the prognosis after treatment is not high compared to other cancers, the patient who undergoes surgery has a better prognosis compared to patients who do not undergo surgery [28]. Patients who developed NOD before PC diagnosis have worse outcomes compared to patients with LTDM or NOD after surgical resection or those who have PC with no DM [29,30]. NOD predicts the most aggressive course of cancer and is considered as one of the strongest prognostic factors to predict the recurrence. NOD reduces the overall survival and disease-free survival in low-tumor (T; T0-T1) stage cancer; however, in one study, there was no significant difference in overall or disease-free survival compared to high T stage cancer [29]. This complex relationship was supported by the hypothesis that the loss of pancreatic parenchyma can cause NOD in aggressive types of PC. Inflammatory and proinflammatory factors could play a role in cell proliferation, migration, and metastasis. However, this finding is not significant and requires further investigation. Mortality after surgery within 90 days are similar in all cohorts of patients (NOD, LTDM, no DM). Patients with LTDM are prone to increased mortality due to related complications [30]. A pooled analysis of three large case-control studies found that PC with neither LTDM nor NOD was associated with short survival. However, when the results are stratified according to the stage of cancer, patients with LTDM accounted for $42 \%$ of the increased death rate in patients with resectable cancer [31].

On the other hand, another study that assessed glucose metabolism by analyzing plasma glucose and HbA1c levels in PC patients after PD showed that $36.8 \%$ of patients resolved their DM after tumor resection and $18 \%$ of patients who were not diabetic preoperatively developed DM [32]. It also explained that insulin resistance improved significantly, but insulin secretion decreased postoperatively. However, it explained that there is an improvement in glucose levels after resection in both PC and non-PC patients. This is explained by the restoration of increased glucagon-like peptide secretion and the stimulating of insulin by preventing fibrosis of the tissue by early resection. But patients undergoing 70-80\% resection of the pancreas had $64.7 \%$ of the incidence of new-onset glucose impairment [33]. However, in their study, PD due to all types of pancreatic pathology was included (chronic pancreatitis, benign and malignant tumors). Patients with prior risk factors like obesity-level BMI (>23 kg/m2) and high cholesterol (>200 mg/dl) are more prone to develop glucose impairment and should be closely monitored for a longer period. Another study showed an incidence of post-surgery glucose impairment/DM of 22\% within a 32-month time period [34]. However, there was a wide range in the incidence of development proposed, which was influenced by the various factors taken into account in the above study. Also, it only included patients with pancreatic ampullary cancer and excluded all other types of cancer. Therefore, more studies are required to test the changes in glucose metabolism in PC with or without DM preoperatively and about the management of impaired glucose levels after PD.

This review has some limitations. We only included studies conducted in humans. Moreover, we only reviewed studies published in English and conducted in the last five years. Perhaps, some potential goodquality papers relevant to this article might have been excluded in the study selection process. Some of the selected studies are observational, and some of them are retrospective. We only focused on the association between type $2 \mathrm{DM}$ and PAC in general, and the association between other types of DM and other PC types was not really part of the scope of our study.

\section{Conclusions}

In this paper, we reviewed the association between DM and PC in the middle-aged and elderly population (>45 years). LTDM is considered to be an independent risk factor for PC development in the elderly. NOD is a consequence and maybe the only early presenting sign of PC. There are several strategies and screening tests proposed that can be used in a stepwise manner in the detection of early-stage PC in NOD patients. A significant association was observed between DM and PC even after the treatment of PC with surgery and such patients should be monitored. However, most of the studies we reviewed were observational, and we believe that more interventional studies are required to further explore this area and to propose definitive early screening tests for PC in NOD and effective methods of postoperative management and follow-up of PC with DM.

\section{Additional Information \\ Disclosures}

Conflicts of interest: In compliance with the ICMJE uniform disclosure form, all authors declare the following: Payment/services info: All authors have declared that no financial support was received from any organization for the submitted work. Financial relationships: All authors have declared that they have no financial relationships at present or within the previous three years with any organizations that might have an interest in the submitted work. Other relationships: All authors have declared that there are no other relationships or activities that could appear to have influenced the submitted work.

\section{References}


1. Maitra A, Sharma A, Brand RE, et al.: A prospective study to establish a new-onset diabetes cohort: from the Consortium for the Study of Chronic Pancreatitis, Diabetes, and Pancreatic Cancer. Pancreas. 2018, 47:1244-1248. 10.1097/MPA.0000000000001169

2. Saeedi P, Petersohn I, Salpea P, et al.: Global and regional diabetes prevalence estimates for 2019 and projections for 2030 and 2045: results from the International Diabetes Federation Diabetes Atlas, 9th Edition. Diabetes Res Clin Pract. 2019, 157:107843. 10.1016/j.diabres.2019.107843

3. LeRoith D, Biessels GJ, Braithwaite SS, et al.: Treatment of diabetes in older adults: an endocrine society clinical practice guideline. J Clin Endocrinol Metab. 2019, 104:1520-1574. 10.1210/jc.2019-00198

4. Woodmansey C, McGovern AP, McCullough KA, et al.: Incidence, demographics, and clinical characteristics of diabetes of the exocrine pancreas (type 3c): a retrospective cohort study. Diabetes Care. 2017, 40:14861493. 10.2337/dc17-0542

5. Song S, Wang B, Zhang X, Hao L, Hu X, Li Z, Sun S: Long-term diabetes mellitus is associated with an increased risk of pancreatic cancer: a meta-analysis. PLoS One. 2015, 10:e0134321. 10.1371/journal.pone.0134321

6. Khadka R, Tian W, Hao X, Koirala R: Risk factor, early diagnosis and overall survival on outcome of association between pancreatic cancer and diabetes mellitus: changes and advances, a review. Int J Surg. 2018, 52:342-346. 10.1016/j.ijsu.2018.02.058

7. Boursi B, Finkelman B, Giantonio BJ, et al.: A clinical prediction model to assess risk for pancreatic cancer among patients with new-onset diabetes. Gastroenterology. 2017, 152:840-850. 10.1053/j.gastro.2016.11.046

8. Lu Y, García Rodríguez LA, Malgerud L, González-Pérez A, Martín-Pérez M, Lagergren J, Bexelius TS: Newonset type 2 diabetes, elevated $\mathrm{HbA1c}$, anti-diabetic medications, and risk of pancreatic cancer. $\mathrm{Br} \mathrm{J}$ Cancer. 2015, 113:1607-1614. 10.1038/bjc.2015.353

9. McWilliams RR, Maisonneuve P, Bamlet WR, et al.: Risk factors for early-onset and very-early-onset pancreatic adenocarcinoma: a Pancreatic Cancer Case-Control Consortium (PanC4) analysis. Pancreas. 2016, 45:311-316. 10.1097/MPA.0000000000000392

10. Škrha P, Hořínek A, Pazourková E, Hajer J, Frič P, Škrha J, Anděl M: Serum microRNA-196 and microRNA200 in pancreatic ductal adenocarcinoma of patients with diabetes mellitus. Pancreatology. 2016, 16:839843. 10.1016/j.pan.2016.05.005

11. Setiawan VW, Stram DO, Porcel J, et al.: Pancreatic cancer following incident diabetes in African Americans and Latinos: the multiethnic cohort. J Natl Cancer Inst. 2019, 111:27-33. 10.1093/jnci/djy090

12. Antolino L, Rocca M, Todde F, et al.: Can pancreatic cancer be detected by adrenomedullin in patients with new-onset diabetes? The PaCANOD cohort study protocol. Tumori. 2018, 104:312-314. 10.5301/tj.5000693

13. Siegel RL, Miller KD, Jemal A: Cancer statistics, 2019. CA Cancer J Clin. 2019, 69:7-34. 10.3322/caac.21551

14. Sharma A, Kandlakunta H, Nagpal SJS, Feng Z, Hoos W, Petersen GM, Chari ST: Model to determine risk of pancreatic cancer in patients with new-onset diabetes. Gastroenterology. 2018, 155:730-739. 10.1053/j.gastro.2018.05.023

15. Kenner BJ, Chari ST, Maitra A, et al.: Early detection of pancreatic cancer-a defined future using lessons from other cancers: a white paper. Pancreas. 2016, 45:1073-1079. 10.1097/MPA.0000000000000701

16. Choe JW, Kim HJ, Kim JS, et al.: Usefulness of CA 19-9 for pancreatic cancer screening in patients with newonset diabetes. Hepatobiliary Pancreat Dis Int. 2018, 17:263-268. 10.1016/j.hbpd.2018.04.001

17. Chari ST, Kelly K, Hollingsworth MA, et al.: Early detection of sporadic pancreatic cancer: summative review. Pancreas. 2015, 44:693-712. 10.1097/MPA.0000000000000368

18. Škrha J, Bušek P, Uhrová J, et al.: Lower plasma levels of glucose-dependent insulinotropic peptide (GIP) and pancreatic polypeptide (PP) in patients with ductal adenocarcinoma of the pancreas and their relation to the presence of impaired glucoregulation and weight loss. Pancreatology. 2017, 17:89-94. 10.1016/j.pan.2016.12.004

19. Kang M, Qin W, Buya M, et al.: VNN1, a potential biomarker for pancreatic cancer-associated new-onset diabetes, aggravates paraneoplastic islet dysfunction by increasing oxidative stress. Cancer Lett. 2016, 373:241-250. 10.1016/j.canlet.2015.12.031

20. He X, Zhong J, Wang S, Zhou Y, Wang L, Zhang Y, Yuan Y: Serum metabolomics differentiating pancreatic cancer from new-onset diabetes. Oncotarget. 2017, 8:29116-29124. 10.18632/oncotarget.16249

21. Mueller AM, Meier CR, Jick SS, Schneider C: Weight change and blood glucose concentration as markers for pancreatic cancer in subjects with new-onset diabetes mellitus: a matched case-control study. Pancreatology. 2019, 19:578-586. 10.1016/j.pan.2019.03.006

22. Dong X, Lou YB, Mu YC, Kang MX, Wu YL: Predictive factors for differentiating pancreatic cancerassociated diabetes mellitus from common type 2 diabetes mellitus for the early detection of pancreatic cancer. Digestion. 2018, 98:209-216. 10.1159/000489169

23. Olson SH, Xu Y, Herzog K, et al.: Weight loss, diabetes, fatigue, and depression preceding pancreatic cancer. Pancreas. 2016, 45:986-991. 10.1097/MPA.0000000000000590

24. Baecker A, Kim S, Risch HA, et al.: Do changes in health reveal the possibility of undiagnosed pancreatic cancer? Development of a risk-prediction model based on healthcare claims data. PLoS One. 2019, 14:e0218580. 10.1371/journal.pone.0218580

25. Illés D, Terzin V, Holzinger G, et al.: New-onset type 2 diabetes mellitus--a high-risk group suitable for the screening of pancreatic cancer?. Pancreatology. 2016, 16:266-271. 10.1016/j.pan.2015.12.005

26. Choe JW, Kim JS, Kim HJ, et al.: Value of early check-up of carbohydrate antigen 19-9 levels for pancreatic cancer screening in asymptomatic new-onset diabetic patients. Pancreas. 2016, 45:730-734. 10.1097/MPA.0000000000000538

27. Dai X, Pang W, Zhou Y, et al.: Altered profile of serum microRNAs in pancreatic cancer-associated newonset diabetes mellitus. J Diabetes. 2016, 8:422-433. 10.1111/1753-0407.12313

28. Fong ZV, Alvino DM, Castillo CF, et al.: Health-related quality of life and functional outcomes in 5-year survivors after pancreaticoduodenectomy. Ann Surg. 2017, 266:685-692. 10.1097/SLA.0000000000002380

29. Lee S, Hwang HK, Kang CM, Lee WJ: Adverse oncologic impact of new-onset diabetes mellitus on recurrence in resected pancreatic ductal adenocarcinoma: a comparison with long-standing and non-diabetes mellitus 


\section{Cureus}

patients. Pancreas. 2018, 47:816-822. 10.1097/MPA.0000000000001099

30. Balzano G, Dugnani E, Gandolfi A, et al.: Effect of diabetes on survival after resection of pancreatic adenocarcinoma. a prospective, observational study. PLoS One. 2016, 11:e0166008.

10.1371/journal.pone.0166008

31. Jeon CY, Li D, Cleary S, et al.: The association of recently diagnosed diabetes and long-term diabetes with survival in pancreatic cancer patients: a pooled analysis. Pancreas. 2018, 47:314-320. 10.1097/MPA.0000000000000989

32. Sohn SY, Lee EK, Han SS, et al.: Favorable glycemic response after pancreatoduodenectomy in both patients with pancreatic cancer and patients with non-pancreatic cancer. Medicine (Baltimore). 2018, $97: \mathrm{e} 0590$. 10.1097/MD.0000000000010590

33. Hwang HK, Park J, Choi SH, Kang CM, Lee WJ: Predicting new-onset diabetes after minimally invasive subtotal distal pancreatectomy in benign and borderline malignant lesions of the pancreas. Medicine (Baltimore). 2017, 96:e9404. 10.1097/MD.0000000000009404

34. Singh AN, Pal S, Kilambi R, Madhusudhan KS, Dash NR, Tandon N, Sahni P: Diabetes after pancreaticoduodenectomy: can we predict it?. J Surg Res. 2018, 227:211-219. 10.1016/j.jss.2018.02.010 